\title{
PERMANENCE AND GLOBAL STABILITY OF POSITIVE \\ SOLUTIONS OF A NONAUTONOMOUS DISCRETE \\ RATIO-DEPENDENT PREDATOR-PREY MODEL
}

\author{
HAI-FENG HUO AND WAN-TONG LI
}

Received 28 October 2004

We first give sufficient conditions for the permanence of nonautonomous discrete ratiodependent predator-prey model. By linearization of the model at positive solutions and construction of Lyapunov function, we also obtain some conditions which ensure that a positive solution of the model is stable and attracts all positive solutions.

\section{Introduction}

In the theoretical ecology, permanence and global stability of the population model are very important. There are extensive literature related to these topics for differential equation models (see $[3,6,7,8,9,12]$ and the references cited therein). Recently, there has been a tendency for some researchers in the field of difference equations to develop some new methods which are analogous to those used in the study of differential equations. (See, e.g., $[1,2,4,5,10,11]$ and the references therein.)

In [5], Fan and Wang considered the following discrete periodic ratio-dependent predator-prey model:

$$
\begin{gathered}
x_{1}(k+1)=x_{1}(k) \exp \left\{a(k)-b(k) x_{1}(k)-\frac{c(k) x_{2}(k)}{m(k) x_{2}(k)+x_{1}(k)}\right\}, \\
x_{2}(k+1)=x_{2}(k) \exp \left\{-d(k)+\frac{f(k) x_{1}(k)}{m(k) x_{2}(k)+x_{1}(k)}\right\},
\end{gathered}
$$

and establish sufficient conditions for the existence of a positive periodic solution of the periodic system (1.1). In this paper, we will establish sufficient conditions for the permanence of system (1.1) and also obtain some conditions which ensure that a positive solution of the model is stable and attracts all positive solutions.

First, we present two definitions.

Definition 1.1. System (1.1) is defined to be permanent if there are positive constants $M$ and $m$ such that each positive solution $\left\{x_{1}(k), x_{2}(k)\right\}$ of system (1.1) satisfies

$$
m \leq \lim \inf _{k \rightarrow \infty} x_{i}(k) \leq \lim \sup _{k \rightarrow \infty} x_{i}(k) \leq M, \quad i=1,2 .
$$


Definition 1.2. System (1.1) is defined to be globally asymptotically stable if a positive solution of system (1.1) is stable and this solution attracts all positive solutions.

Throughout this paper, we will assume that $a(k), b(k), c(k), d(k), m(k)$, and $f(k)$ are bounded nonnegative sequences, and use the following notations: for any bounded sequence $\{u(k)\}$,

$$
u^{M}=\sup _{k \in \mathbb{N}} u(k), \quad u^{L}=\inf _{k \in \mathbb{N}} u(k)
$$

For biological reasons, we only consider solution $\left\{x_{1}(k), x_{2}(k)\right\}$, with $x_{1}(0)>0, x_{2}(0)>0$.

The organization of this paper is the following. In the next section, we establish the permanence of system (1.1). In Section 3, we obtain the sufficient conditions which ensure that a positive solution of system (1.1) is stable and attracts all positive solutions.

\section{Permanence}

In this section, we establish a permanence result for system (1.1).

Lemma 2.1. For every solution $\left\{x_{1}(k), x_{2}(k)\right\}$ of (1.1),

$$
\lim _{k \rightarrow \infty} \sup x_{1}(k) \leq B_{1}, \quad \lim _{k \rightarrow \infty} \sup x_{2}(k) \leq B_{2},
$$

where

$$
B_{1}=\max \left\{\frac{a^{M}}{b^{L}}, \frac{\exp \left(a^{M}-1\right)}{b^{L}}\right\}, \quad B_{2}=\left\{\frac{f^{M} B_{1}}{m^{L} d^{L}}, \frac{f^{M} B_{1}}{m^{L} d^{L}} \exp \left\{-d^{L}+f^{M}\right\}\right\} .
$$

Proof. Clearly, $x_{1}(k)>0$ and $x_{2}(k)>0$ for $k \geq 0$. We first prove that

$$
\lim _{k \rightarrow \infty} \sup x_{1}(k) \leq B_{1} .
$$

To prove (2.3), we first assume that there exists an $l_{0} \in \mathbb{N}$ such that $x_{1}\left(l_{0}+1\right) \geq x_{1}\left(l_{0}\right)$. Then,

$$
a\left(l_{0}\right)-b\left(l_{0}\right) x_{1}\left(l_{0}\right)-\frac{c\left(l_{0}\right) x_{2}\left(l_{0}\right)}{m\left(l_{0}\right) x_{2}\left(l_{0}\right)+x_{1}\left(l_{0}\right)} \geq 0
$$

Hence,

$$
x_{1}\left(l_{0}\right) \leq \frac{a\left(l_{0}\right)}{b\left(l_{0}\right)} \leq \frac{a^{M}}{b^{L}}
$$

It follows that

$$
\begin{aligned}
x_{1}\left(l_{0}+1\right) & =x_{1}\left(l_{0}\right) \exp \left\{a\left(l_{0}\right)-b\left(l_{0}\right) x_{1}\left(l_{0}\right)-\frac{c\left(l_{0}\right) x_{2}\left(l_{0}\right)}{m\left(l_{0}\right) x_{2}\left(l_{0}\right)+x_{1}\left(l_{0}\right)}\right\} \\
& \leq x_{1}\left(l_{0}\right) \exp \left\{a\left(l_{0}\right)-b\left(l_{0}\right) x_{1}\left(l_{0}\right)\right\} \\
& \leq x_{1}\left(l_{0}\right) \exp \left\{a^{M}-b^{L} x_{1}\left(l_{0}\right)\right\} \leq \frac{\exp \left(a^{M}-1\right)}{b^{L}},
\end{aligned}
$$


here we used

$$
\max _{x \in \mathbb{R}}\{x \exp (a-b x)\}=\frac{\exp (a-1)}{b}, \quad \text { for } a, b>0
$$

We claim that

$$
x_{1}(k) \leq B_{1}, \quad \text { for } k \geq l_{0} .
$$

By way of contradiction, assume that there exists a $p_{0}>l_{0}$ such that $x_{1}\left(p_{0}\right)>B_{1}$. Then $p_{0} \geq l_{0}+2$. Let $\tilde{p}_{0} \geq l_{0}+2$ be the smallest integer such that $x_{1}\left(\tilde{p}_{0}\right)>B_{1}$. Then $x_{1}\left(\tilde{p}_{0-1}\right)<$ $x_{1}\left(\tilde{p}_{0}\right)$. The above argument produces that $x_{1}\left(\tilde{p}_{0}\right) \leq B_{1}$, a contradiction. This proves the claim. Now, we assume that $x_{1}(k+1)<x_{1}(k)$ for all $k \in \mathbb{N}$. In particular, $\lim _{k \rightarrow \infty} x_{1}(k)$ exists, denoted by $\bar{x}_{1}$. We claim that $\bar{x}_{1} \leq a^{M} / b^{L}$. By way of contradiction, assume that $\bar{x}_{1}>a^{M} / b^{L}$. Taking limit in the first equation in system (1.1) gives

$$
\lim _{k \rightarrow \infty}\left(a(k)-b(k) x_{1}(k)-\frac{c(k) x_{2}(k)}{m(k) x_{2}(k)+x_{1}(k)}\right)=0,
$$

which is a contradiction since

$$
a(k)-b(k) x_{1}(k)-\frac{c(k) x_{2}(k)}{m(k) x_{2}(k)+x_{1}(k)} \leq a(k)-b(k) x_{1}(k) \leq a^{M}-b^{L} \bar{x}_{1}<0, \quad \text { for } n \in \mathbb{N} .
$$

This proves the claim. Note that $a^{M} / b^{L} \leq B_{1}$. It follows that (2.3) holds.

Next, we prove that

$$
\lim _{k \rightarrow \infty} \sup _{2}(k) \leq B_{2}
$$

At first, we assume that there exists an $n_{0} \in \mathbb{N}$ such that $x_{2}\left(n_{0}+1\right) \geq x_{2}\left(n_{0}\right)$. Then

$$
-d\left(n_{0}\right)+\frac{f\left(n_{0}\right) x_{1}\left(n_{0}\right)}{m\left(n_{0}\right) x_{2}\left(n_{0}\right)+x_{1}\left(n_{0}\right)} \geq 0 .
$$

Hence,

$$
\begin{gathered}
-d\left(n_{0}\right)+\frac{f\left(n_{0}\right) x_{1}\left(n_{0}\right)}{m\left(n_{0}\right) x_{2}\left(n_{0}\right)} \geq 0, \\
x_{2}\left(n_{0}\right) \leq \frac{f\left(n_{0}\right) x_{1}\left(n_{0}\right)}{m\left(n_{0}\right) d\left(n_{0}\right)} \leq \frac{f^{M} B_{1}}{m^{L} d^{L}} .
\end{gathered}
$$

It follows that

$$
\begin{aligned}
x_{2}\left(n_{0}+1\right) & =x_{2}\left(n_{0}\right) \exp \left\{-d\left(n_{0}\right)+\frac{f\left(n_{0}\right) x_{1}\left(n_{0}\right)}{m\left(n_{0}\right) x_{2}\left(n_{0}\right)+x_{1}\left(n_{0}\right)}\right\} \\
& \leq \frac{f^{M} B_{1}}{m^{L} d^{L}} \exp \left\{-d^{L}+f^{M}\right\} .
\end{aligned}
$$


We claim that $x_{2}(k) \leq B_{2}$ for $k \geq n_{0}$. By way of contradiction, assume that there exists a $q_{0}>n_{0}$ such that $x_{2}\left(q_{0}\right)>B_{2}$. Then $q_{0}=n_{0}+2$. Let $\tilde{q}_{0} \geq n_{0}+2$ be the smallest integer such that $x_{2}\left(\tilde{q}_{0}\right)>B_{2}$. Then $x_{2}\left(\tilde{q}_{0-1}\right)<x_{2}\left(\tilde{q}_{0}\right)$. The above argument produces that $x_{2}\left(\tilde{q}_{0}\right) \leq B_{2}$, a contradiction. This proves the claim. Now, we assume that $x_{2}(k+1)<x_{2}(k)$ for all $k \in$ N. In particular, $\lim _{k \rightarrow \infty} x_{2}(k)$ exists, denoted by $\bar{x}_{2}$. We claim that $\bar{x}_{2} \leq f^{M} B_{1} / m^{L} d^{L}$. By way of contradiction, assume that $\bar{x}_{2}>f^{M} B_{1} / m^{L} d^{L}$. Taking limit in the second equation in system (1.1) gives

$$
\lim _{k \rightarrow \infty}\left(-d(k)+\frac{f(k) x_{1}(k)}{m(k) x_{2}(k)+x_{1}(k)}\right)=0,
$$

which is a contradiction since

$$
-d(k)+\frac{f(k) x_{1}(k)}{m(k) x_{2}(k)+x_{1}(k)} \leq-d^{L}+\frac{f^{M} B_{1}}{m^{L} \bar{x}_{2}}<0 .
$$

It follows that (2.11) holds. This completes the proof.

Lemma 2.2. Assume that

$$
a^{L}>\frac{c^{M}}{m^{L}}, \quad f^{L}>d^{M}
$$

Then

$$
\lim \inf _{k \rightarrow \infty} x_{1}(k) \geq D_{1}, \quad \lim \inf _{k \rightarrow \infty} x_{2}(k) \geq D_{2},
$$

where

$$
\begin{aligned}
& D_{1}=\min \left\{\frac{a^{L}-c^{M} / m^{L}}{b^{M}} \exp \left\{a^{L}-b^{M} B_{1}-\frac{c^{M}}{m^{L}}\right\}, \frac{a^{L}-c^{M} / m^{L}}{b^{M}}\right\}, \\
& D_{2}=\min \left\{\frac{f^{L}-d^{M}}{m^{M} d^{M}} D_{1}, \frac{f^{L}-d^{M}}{m^{M} d^{M}} \exp \left\{-d^{M}+\frac{f^{L} D_{1}}{m^{M} B_{2}+D_{1}}\right\}\right\} .
\end{aligned}
$$

Proof. We first show that

$$
\lim \inf _{k \rightarrow \infty} x_{1}(k) \geq D_{1}
$$

According to Lemma 2.1, there exists a $k^{*} \in \mathbb{N}$ such that

$$
x_{1}(k) \leq B_{1}+\epsilon, \quad x_{2}(k) \leq B_{2}+\epsilon, \quad \text { for } k \geq k^{*} .
$$


Firstly, we assume that there exists an $l_{0} \geq k^{*}$ such that $x_{1}\left(l_{0}+1\right) \leq x_{1}\left(l_{0}\right)$. Note that, for $k \geq l_{0}$,

$$
\begin{aligned}
x_{1}(k+1) & =x_{1}(k) \exp \left\{a(k)-b(k) x_{1}(k)-\frac{c(k) x_{2}(k)}{m(k) x_{2}(k)+x_{1}(k)}\right\} \\
& \geq x_{1}(k) \exp \left\{a(k)-b(k) x_{1}(k)-\frac{c(k)}{m(k)}\right\} \\
& \geq x_{1}(k) \exp \left\{a^{L}-b^{M} x_{1}(k)-\frac{c^{M}}{m^{L}}\right\} .
\end{aligned}
$$

In particular, with $k=l_{0}$, we have

$$
a^{L}-b^{M} x_{1}\left(l_{0}\right)-\frac{c^{M}}{m^{L}} \leq 0
$$

which implies that

$$
x_{1}\left(l_{0}\right) \geq \frac{a^{L}-c^{M} / m^{L}}{b^{M}}
$$

Then

$$
x_{1}\left(l_{0}+1\right) \geq \frac{a^{L}-c^{M} / m^{L}}{b^{M}} \exp \left\{a^{L}-b^{M}\left(B_{1}+\epsilon\right)-\frac{c^{M}}{m^{L}}\right\} .
$$

Let

$$
x_{1 \epsilon}=\frac{a^{L}-c^{M} / m^{L}}{b^{M}} \exp \left\{a^{L}-b^{M}\left(B_{1}+\epsilon\right)-\frac{c^{M}}{m^{L}}\right\} .
$$

We claim that

$$
x_{1}(k) \leq x_{1 \epsilon}, \quad \text { for } k \geq l_{0} .
$$

By way of contradiction, assume that there exists a $p_{0} \geq l_{0}$ such that $x_{1}\left(p_{0}\right)<x_{1 \epsilon}$. Then $p_{0} \geq l_{0}+2$. Let $\tilde{p}_{0} \geq l_{0}+2$ be the smallest integer such that $x_{1}\left(\tilde{p}_{0}\right)<x_{1 \epsilon}$. Then $x_{1}\left(\tilde{p}_{0}-1\right)>$ $x_{1}\left(\tilde{p}_{0}\right)$. The above argument produces that $x_{1}\left(\tilde{p}_{0}\right) \geq x_{1 \epsilon}$, a contradiction. This proves the claim. Now, we assume that $x_{1}(k+1)>x_{1}(k)$ for all $k \in \mathbb{N}$. In particular, $\lim _{k \rightarrow \infty} x_{1}(k)$ exists, denoted by $\underline{x}_{1}$. We claim that

$$
\underline{x}_{1} \geq \frac{\left(a^{L}-c^{M} / m^{L}\right)}{b^{M}} .
$$

By way of contradiction, assume that

$$
\underline{x}_{1}<\frac{\left(a^{L}-c^{M} / m^{L}\right)}{b^{M}} .
$$


Taking limit in the first equation in system (1.1) gives

$$
\lim _{k \rightarrow \infty}\left(a(k)-b(k) x_{1}(k)-\frac{c(k) x_{2}(k)}{m(k) x_{2}(k)+x_{1}(k)}\right)=0,
$$

which is a contradiction since

$$
\lim _{k \rightarrow \infty}\left(a(k)-b(k) x_{1}(k)-\frac{c(k) x_{2}(k)}{m(k) x_{2}(k)+x_{1}(k)}\right) \geq a^{L}-b^{M} \underline{x}_{1}-\frac{c^{M}}{m^{L}}>0 .
$$

This proves the claim. It follows that (2.20) holds.

Next, we prove that

$$
\lim \inf _{k \rightarrow \infty} x_{2}(k) \geq D_{2} .
$$

At first, we assume that there exists an $n_{0} \in \mathbb{N}$ such that $x_{2}\left(n_{0}+1\right) \geq x_{2}\left(n_{0}\right)$. Note that, for $k \geq n_{0}$,

$$
\begin{aligned}
x_{2}(k+1) & =x_{2}(k) \exp \left\{-d(k)+\frac{f(k) x_{1}(k)}{m(k) x_{2}(k)+x_{1}(k)}\right\} \\
& \geq x_{2}(k) \exp \left\{-d(k)+\frac{f(k) D_{1}}{m(k) x_{2}(k)+D_{1}}\right\} .
\end{aligned}
$$

In particular, with $k=n_{0}$, we get

$$
-d\left(n_{0}\right)+\frac{f\left(n_{0}\right) D_{1}}{m\left(n_{0}\right) x_{2}\left(n_{0}\right)+D_{1}} \leq 0,
$$

which implies that

$$
x_{2}\left(n_{0}\right) \geq \frac{f^{L}-d^{M}}{m^{M} d^{M}} D_{1}
$$

Then

$$
x_{2}\left(n_{0}+1\right) \geq \frac{f^{L}-d^{M}}{m^{M} d^{M}} D_{1} \exp \left\{-d^{M}+\frac{f^{L} D_{1}}{m^{M}\left(B_{2}+\epsilon\right)+D_{1}}\right\} .
$$

Let

$$
x_{2 \epsilon}=\frac{f^{L}-d^{M}}{m^{M} d^{M}} D_{1} \exp \left\{-d^{M}+\frac{f^{L} D_{1}}{m^{M}\left(B_{2}+\epsilon\right)+D_{1}}\right\} .
$$

We claim that $x_{2}(k) \geq x_{2 \epsilon}$ for $k \geq n_{0}$. By way of contradiction, assume that there exists a $q_{0} \geq n_{0}$ such that $x_{2}\left(q_{0}\right)<x_{2 \epsilon}$. Then $q_{0} \geq n_{0}+2$. Let $\tilde{q}_{0} \geq n_{0}+2$ be the smallest integer such that $x_{2}\left(\tilde{q}_{0}\right)<x_{2 \epsilon}$. Then $x_{2}\left(\tilde{q}_{0}-1\right)>x_{2}\left(\tilde{q}_{0}\right)$. The above argument produces that $x_{2}\left(\tilde{q}_{0}\right) \geq x_{2 \epsilon}$, a contradiction. This proves the claim. Now, we assume that $x_{2}(k+1)<$ $x_{2}(k)$ for all $k \in \mathbb{N}$. In particular, $\lim _{k \rightarrow \infty} x_{2}(k)$ exists, denoted by $\underline{x}_{2}$. We claim that

$$
\underline{x}_{2} \geq \frac{f^{L}-d^{M}}{m^{M} d^{M}} D_{1}
$$


By way of contradiction, assume that

$$
\underline{x}_{2}<\frac{f^{L}-d^{M}}{m^{M} d^{M}} D_{1}
$$

Taking limit in the second equation in system (1.1) gives

$$
\lim _{k \rightarrow \infty}\left(-d(k)+\frac{f(k) x_{1}(k)}{m(k) x_{2}(k)+x_{1}(k)}\right)=0,
$$

which is a contradiction since

$$
-d(k)+\frac{f(k) x_{1}(k)}{m(k) x_{2}(k)+x_{1}(k)} \geq-d^{M}+\frac{f^{L} D_{1}}{m^{M} \underline{x}_{2}+D_{1}}>0 .
$$

It follows that (2.32) holds. This completes the proof.

Now, by Lemmas 2.1 and 2.2, we can easily obtain the following result.

Theorem 2.3. Assume that

$$
a^{L}>\frac{c^{M}}{m^{L}}, \quad f^{L}>d^{M}
$$

Then system (1.1) is permanent.

\section{Global stability}

In this section, we derive sufficient conditions which guarantee that the positive solution of (1.1) is globally stable. Our strategy in the proof of the global stability of the positive solution of (1.1) is to construct suitable Lyapunov functions

Theorem 3.1. In addition to the assumptions made in Theorem 2.3, assume further that

(i) there exist positive constant $v$ and positive constants $n_{i}, i=1,2$, such that

$$
\min \left\{n_{1} b(k)-n_{1} \frac{c(k)}{4 m(k) D_{1}}-n_{2} \frac{f(k)}{4 D_{1}}, n_{2} \frac{f(k) D_{1}}{\left(m(k) B_{2}+B_{1}\right)^{2}}-n_{1} \frac{c(k)}{4 m(k) D_{2}}\right\}>v
$$

for all large $k$, where $D_{i}$ and $B_{i}$ are given in Lemmas 2.1 and 2.2,

(ii) $b(k) B_{1} \leq 1$ and $f(k) \leq 4$ for all large $k$, where $B_{1}$ is given in Lemma 2.1.

Then system (1.1) is globally asymptotically stable, that is, a positive solution of (1.1) is stable and attracts all positive solutions.

Proof. Let $\left\{x_{1}^{*}(k), x_{2}^{*}(k)\right\}$ be a positive solution of (1.1). We prove below that it is uniformly asymptotically stable. To this end, we introduce the change of variables

$$
u_{1}(k)=x_{1}(k)-x_{1}^{*}(k), \quad u_{2}(k)=x_{2}(k)-x_{2}^{*}(k)
$$


System (1.1) is then transformed into

$$
\begin{aligned}
u_{1}(k+1)= & x_{1}(k) \exp \left\{a(k)-b(k) x_{1}(k)-\frac{c(k) x_{1}(k) x_{2}(k)}{m(k) x_{2}(k)+x_{1}(k)}\right\} \\
& -x_{1}^{*}(k) \exp \left\{a(k)-b(k) x_{1}^{*}(k)-\frac{c(k) x_{1}^{*}(k) x_{2}^{*}(k)}{a_{1}(k) x_{2}^{*}(k)+x_{1}^{*}(k)}\right\}, \\
u_{2}(k+1)= & x_{2}(k) \exp \left\{\frac{f(k) x_{1}(k)}{m(k) x_{2}(k)+x_{1}(k)}-d(k)\right\} \\
& -x_{2}^{*}(k) \exp \left\{\frac{f(k) x_{1}^{*}(k)}{a_{1}(k) x_{2}^{*}(k)+x_{1}^{*}(k)}-d(k)\right\}
\end{aligned}
$$

which, by Taylor formula, can be rewritten as

$$
\begin{aligned}
& u_{1}(k+1)= \exp \left\{a(k)-b(k) x_{1}^{*}(k)-\frac{c(k) x_{1}^{*}(k) x_{2}^{*}(k)}{m(k) x_{2}^{*}(k)+x_{1}^{*}(k)}\right\} \\
& \times\left(1-b(k) x_{1}^{*}(k)+\frac{c(k) x_{1}^{*}(k) x_{2}^{*}(k)}{\left(m(k) x_{2}^{*}(k)+x_{1}^{*}(k)\right)^{2}}\right) u_{1}(k) \\
&\left.-\frac{c(k) x_{1}^{*}(k)}{\left(m(k) x_{2}^{*}(k)+x_{1}^{*}(k)\right)^{2}} x_{1}^{*}(k) u_{2}(k)+f_{1}(k, u(k))\right), \\
& u_{2}(k+1)=\exp \left\{\frac{f(k) x_{1}^{*}(k)}{m(k) x_{2}^{*}(k)+x_{1}^{*}(k)}-d(k)\right\} \\
& \times\left(\left(1-\frac{f(k) m(k) x_{1}^{*}(k) x_{2}^{*}(k)}{\left(m(k) x_{2}^{*}(k)+x_{1}^{*}(k)\right)^{2}}\right) u_{2}(k)\right. \\
&\left.\quad+\frac{f(k) m(k)\left(x_{2}^{*}(k)\right)^{2}}{\left(m(k) x_{2}^{*}(k)+x_{1}^{*}(k)\right)^{2}} u_{1}(k)+f_{2}(k, u(k))\right),
\end{aligned}
$$

where $\left|f_{i}(k, u)\right| /\|u\|$ converges, uniformly with respect to $k \in \mathbb{N}$, to zero as $\|u\| \rightarrow 0$. In view of system (1.1), it follows from (3.4) that

$$
\begin{aligned}
u_{1}(k+1)=x_{1}^{*}(k+1)( & \left(1-b(k) x_{1}^{*}(k)+\frac{c(k) x_{1}^{*}(k) x_{2}^{*}(k)}{\left(m(k) x_{2}^{*}(k)+x_{1}^{*}(k)\right)^{2}}\right) \frac{u_{1}(k)}{x_{1}^{*}(k)} \\
& \left.-\frac{c(k) x_{1}^{*}(k)}{\left(m(k) x_{2}^{*}(k)+x_{1}^{*}(k)\right)^{2}} u_{2}(k)+\frac{f_{1}(k, u(k))}{x_{1}^{*}(k)}\right), \\
u_{2}(k+1)=x_{2}^{*}(k+1)( & \left(1-\frac{f(k) m(k) x_{1}^{*}(k) x_{2}^{*}(k)}{\left(m(k) x_{2}^{*}(k)+x_{1}^{*}(k)\right)^{2}}\right) \frac{u_{2}(k)}{x_{2}^{*}(k)} \\
& \left.+\frac{f(k) m(k) x_{2}^{*}(k)}{\left(m(k) x_{2}^{*}(k)+x_{1}^{*}(k)\right)^{2}} u_{1}(k)+\frac{f_{2}(k, u(k))}{x_{2}^{*}(k)}\right),
\end{aligned}
$$


where $\left|f_{i}(k, u)\right| /\|u\|$ converges, uniformly with respect to $k \in \mathbb{N}$, to zero as $\|u\| \rightarrow 0$. We define the function $V$ by

$$
V(u(k))=n_{1}\left|\frac{u_{1}(k)}{x_{1}^{*}(k)}\right|+n_{2}\left|\frac{u_{2}(k)}{x_{2}^{*}(k)}\right|,
$$

where $n_{j}$ are positive constants given in (i). Calculating the difference of $V$ along the solution of system (3.5) and using (ii), we obtain

$$
\begin{aligned}
\Delta V \leq & -\left(n_{1} b(k)-n_{1} \frac{c(k)}{4 m(k) D_{1}}-n_{2} \frac{f(k)}{4 D_{1}}\right) x_{1}^{*}(k)\left|\frac{u_{1}(k)}{x_{1}^{*}(k)}\right| \\
& -\left(n_{2} \frac{f(k) D_{1}}{\left(m(k) B_{2}+B_{1}\right)^{2}}-n_{1} \frac{c(k)}{4 m(k) D_{2}}\right) x_{2}^{*}(k)\left|\frac{u_{2}(k)}{x_{2}^{*}(k)}\right| \\
& +n_{1} \frac{\left|f_{1}(k, u(k))\right|}{x_{1}^{*}(k)}+n_{2} \frac{\left|f_{1}(k, u(k))\right|}{x_{2}^{*}(k)}, \text { for large } k .
\end{aligned}
$$

Since $\left|f_{i}(k, u)\right| /\|u\|$ converges uniformly to zero as $\|u\| \rightarrow 0$, it follows from condition (i) and Theorem 2.3 that there is a positive constant $\gamma$ such that if $k$ is sufficiently large and $\|u(k)\|<\gamma$,

$$
\Delta V \leq-\frac{v\|u(k)\|}{2}
$$

By [1], we see that the trivial solution of (3.5) is uniformly asymptotically stable, and so is the solution $\left\{x_{1}^{*}(k), x_{2}^{*}(k)\right\}$ of $(1.1)$. Note that the positive solution $\left\{x_{1}(k), x_{2}(k)\right\}$ is chosen in an arbitrary way. Proceeding exactly as in [11], we conclude that the positive solution $\left\{x_{1}^{*}(k), x_{2}^{*}(k)\right\}$ of $(1.1)$ is globally stable. The proof is complete

\section{Acknowledgments}

The first author was supported by the NSF of Gansu Province of China, the NSF of Bureau of Education of Gansu Province of China (0416B-08), the Key Research and Development Program for Outstanding Groups of Lanzhou University of Technology, and the Development Program for Outstanding Young Teachers in Lanzhou University of Technology. The second author was supported by the NNSF of China (10171040), the NSF of Gansu Province of China (ZS011-A25-007-Z), and the Teaching and Research Award Program for Outstanding Young Teachers in Higher Education Institutions of the Ministry of Education of China.

\section{References}

[1] R. P. Agarwal, Difference Equations and Inequalities. Theory, Methods, and Applications, 2nd ed., Monographs and Textbooks in Pure and Applied Mathematics, vol. 228, Marcel Dekker, New York, 2000.

[2] R. P. Agarwal and P. J. Y. Wong, Advanced Topics in Difference Equations, Mathematics and Its Applications, vol. 404, Kluwer Academic, Dordrecht, 1997. 


\section{Discrete ratio-dependent predator-prey model}

[3] L. S. Chen, Z. Y. Lu, and W. D. Wang, The effect of delays on the permanence for Lotka-Volterra systems, Appl. Math. Lett. 8 (1995), no. 4, 71-73.

[4] Y. M. Chen and Z. Zhou, Stable periodic solution of a discrete periodic Lotka-Volterra competition system, J. Math. Anal. Appl. 277 (2003), no. 1, 358-366.

[5] M. Fan and K. Wang, Periodic solutions of a discrete time nonautonomous ratio-dependent predator-prey system, Math. Comput. Modelling 35 (2002), no. 9-10, 951-961.

[6] H. I. Freedman and S. G. Ruan, Uniform persistence in functional-differential equations, J. Differential Equations 115 (1995), no. 1, 173-192.

[7] H.-F. Huo, W.-T. Li, and S. S. Cheng, Periodic solutions of two-species diffusion models with continuous time delays, Demonstratio Math. 35 (2002), no. 2, 433-446.

[8] Y. Kuang, Delay Differential Equations with Applications in Population Dynamics, Mathematics in Science and Engineering, vol. 191, Academic Press, Massachusetts, 1993.

[9] Z. Y. Lu and Y. Takeuchi, Permanence and global attractivity for competitive Lotka-Volterra systems with delay, Nonlinear Anal. 22 (1994), no. 7, 847-856.

[10] Y. Muroya, Persistence and global stability for discrete models of nonautonomous Lotka-Volterra type, J. Math. Anal. Appl. 273 (2002), no. 2, 492-511.

[11] W. Wendi and L. Zhengyi, Global stability of discrete models of Lotka-Volterra type, Nonlinear Anal. Ser. B: Real World Applications 35 (1999), no. 8, 1019-1030.

[12] W. Wendi and M. Zhien, Harmless delays for uniform persistence, J. Math. Anal. Appl. 158 (1991), no. 1, 256-268.

Hai-Feng Huo: Department of Applied Mathematics, Lanzhou University of Technology, Lanzhou, Gansu 730050, China

E-mail address: hfhuo@lut.cn

Wan-Tong Li: Department of Mathematics, Lanzhou University, Lanzhou, Gansu 730000, China

E-mail address: wtli@lzu.edu.cn 


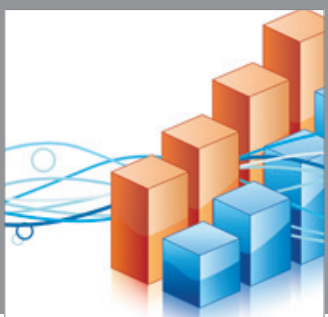

Advances in

Operations Research

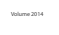

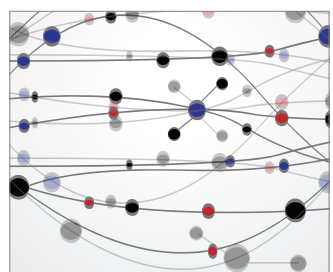

\section{The Scientific} World Journal
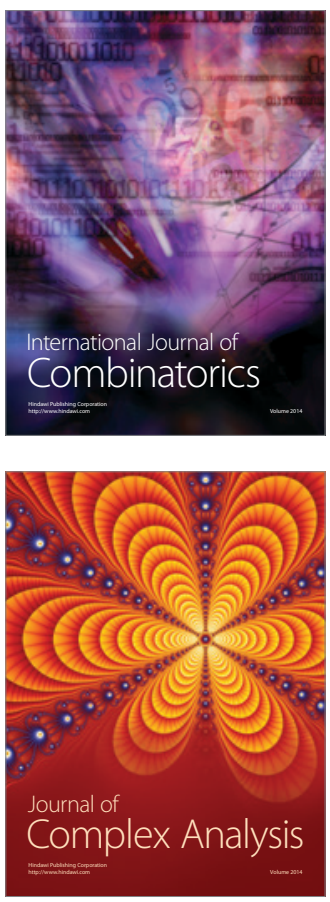

International Journal of

Mathematics and

Mathematical

Sciences
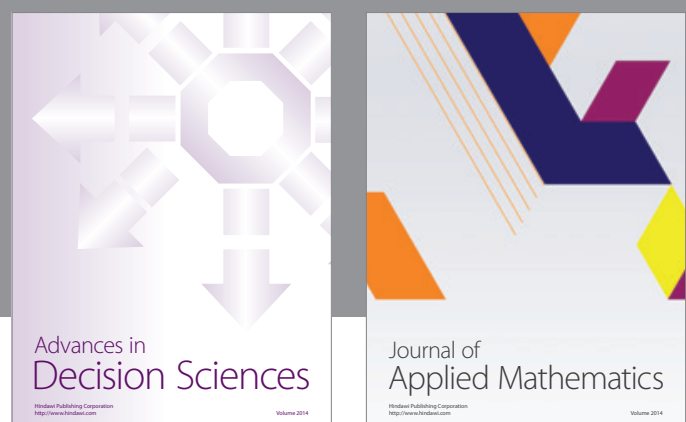

Journal of

Applied Mathematics
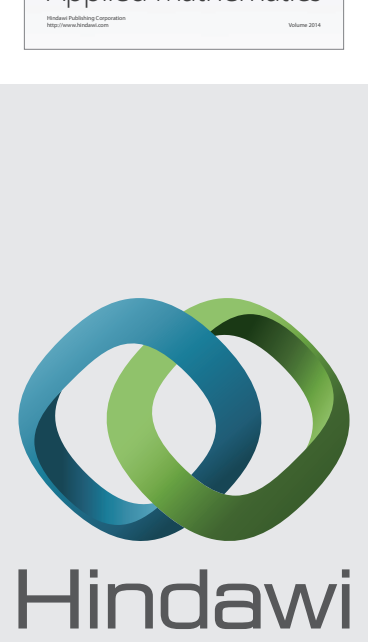

Submit your manuscripts at http://www.hindawi.com
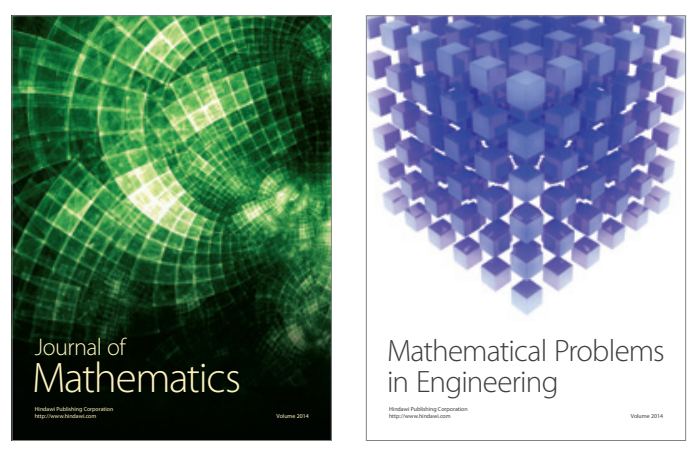

Mathematical Problems in Engineering
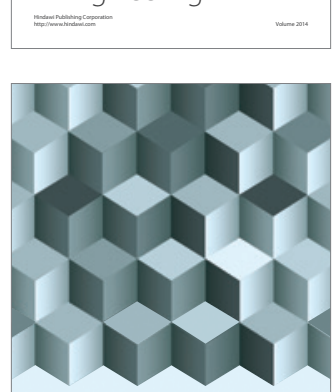

Journal of

Function Spaces
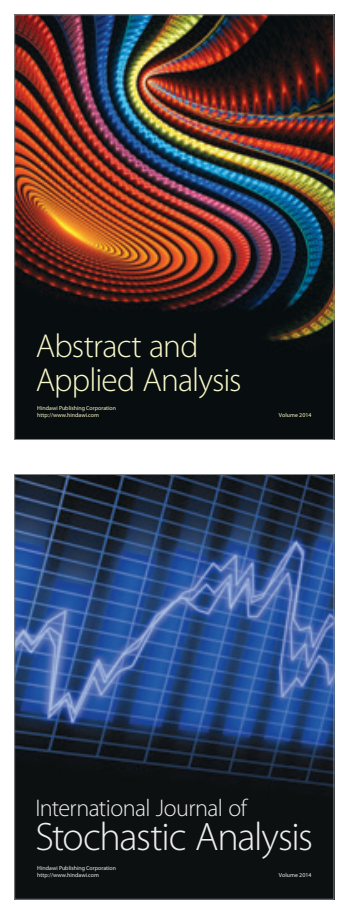

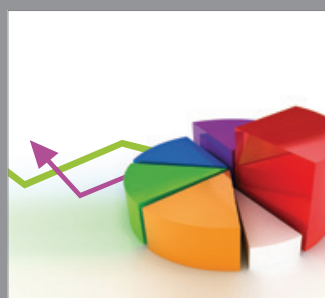

ournal of

Probability and Statistics

Promensencen
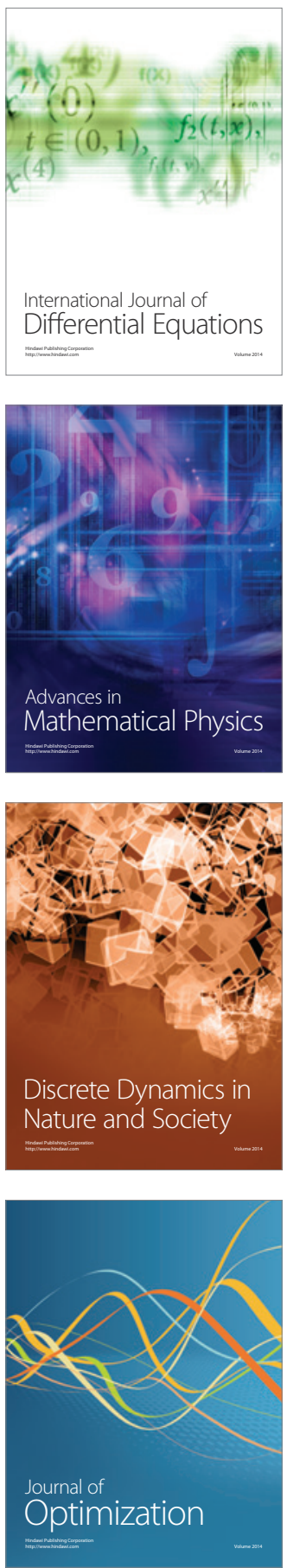